\title{
A Novel Semi-Blind Reference Color Image Watermarking using DWT-DCT-SVD
}

\author{
Satyanarayana Murty.P \\ Research Scholar \\ Department of ECE \\ AU College of Engg \\ Andhra University
}

\author{
Hima Bindu. R \\ UG Student \\ Department of ECE \\ GIITS College of Engg
}

\author{
Rajesh Kumar. P \\ Associate Professor \\ Department of ECE \\ AU College of Engg \\ Andhra University
}

\begin{abstract}
In this paper we propose three robust and semi-blind digital color image watermarking algorithms. These algorithms are based on hybrid transforms using the combination of Discrete Cosine Transform (DCT) and Singular Value Decomposition (SVD), Discrete Wavelet Transform (DWT) and Singular Value Decomposition (SVD), Discrete Wavelet Transform (DWT), Discrete Cosine Transform (DCT) and Singular Value Decomposition (SVD). The original color image is divided to number of blocks. We calculate the spatial frequency of each block. We kept a threshold on this spatial frequency and from this we form a reference image. In the first algorithm we apply DCT on reference image. The singular values of the applied DCT coefficients and singular values of watermark are modified. In the second algorithm we apply DWT on reference image. Then the reference image divided into four sub bands called LL, LH, HL and HH. The singular values of LL band and singular values of watermark are modified. In third algorithm we apply DWT on reference image. Then the reference image divided into four sub bands called LL, LH, HL and HH. We apply DCT on LL band. The singular values of the applied DCT coefficients and singular values of watermark are modified. The performance of the proposed algorithms was evaluated with respect to imperceptibility. The three algorithms are provided almost good imperceptibility and the robustness has varied against various attacks.
\end{abstract}

\section{Keywords}

Network, Storage Devices, multimedia, watermarking, DCT, DWT, SVD, Robustness

\section{INTRODUCTION}

Due to the rapid and extensive growth of network technology, digital information can now be distributed much faster and easier. However, according to the insufficient cognizance of intellectual property, the condition of illegal copies and spread of copyright reserved information are growing serious. To protect the copyright of multimedia information and to decrease the impulse to copy and spread copy right reserved multimedia information. There are immense technical challenges in discouraging unauthorized copying and distributing of digital information. Fortunately, digital watermarking technique has been proposed as a method to embed an invisible signal into multimedia data so as to attest the owner identification of the data and discourage the unauthorized copying and distributing of digital information. In digital image watermarking the inserted watermark should not degrade the visual perception of an original image. This information of digital data can be extracted later for ownership verification [1]. Digital watermarking can applied to a variety of fields like text, image, audio, video and software. A lot of techniques are available for protecting the copyrighted material. The first method for hiding watermarking is by directly changing original cover-media. The advantages are simple and fast calculated but cannot protect itself from varied signal processing attacking [2, 3]. The most of watermarking techniques embed the information data in the coefficients of transformation domain of the cover image, such as Fourier transformation, discrete cosine transformation, wavelet transformation and singular value decomposition. Image watermarking algorithms using Discrete Cosine Transform (DCT) [4], Discrete Wavelet Transform (DWT) [5], and Singular Value Decomposition (SVD) [6] are available in the literature. Domain transformation watermarking schemes, in general, first use DCT and DWT and then transforms the image into the spatial domain. Watermarking schemes usually focus on watermarking black and white or grayscale images. The data hiding capacity is high in spatial domain and frequency domain algorithms based on DCT, SVD. However, these algorithms are hardly robust against various attacks, prone to tamper and degrade the quality of the watermarked image. Hybrid domain transforms are also available in the literature DCT- SVD [7] and DWT-SVD [8].

In this paper we proposed three semi - blind color image watermarking algorithms using DCT- SVD, DWTSVD and DWT-DCT-SVD schemes. The rest of the paper is organized as follows: Section 2 describes related work while Section 3 provides our proposed algorithms, section 4 experimental results and in section 5 conclusions and in Section 6 references.

\section{RELATED WORK}

Authors proposed a hybrid algorithm for color image watermarking [11]. They tested their proposed method for binary, grayscale and color watermark images. They used contourlet transform and singular value decomposition to embed the watermark. They divided the color image into RED, GREEN and BLUE color planes. The singular values of mid frequency sub-band coefficients of color watermark image are embedded into singular values of mid frequency 
sub-band coefficients of host color image in Red, Green and Blue color spaces simultaneously based on spread spectrum technique. The robustness of watermark is improved for common image procession operations by combining both the concepts of contourlet transform and singular value decomposition.

Authors propose [12] a digital watermarking method for color images based on wavelet transform. In the proposed method, the authors embed a watermark into both the luminance component and the chrominance component by using two different algorithms. In luminance component the watermark embedded by dwt, while in chrominance component by relations between neighboring coefficients.

Authors present [13] a more secure method for copyright protection. In this scheme color image is decomposed into R, G, B channels and then DWT and DCT transformations are applied on these channels separately. The bits of watermark image are embedded into middle frequency coefficients of transformed R, G, B channels.

Basically here also the authors proposed [14] a color image watermarking using DWT-DCT. The color image was decomposed into YIQ format. The security levels are increased by using multiple pn sequences, Arnold scrambling, DWT domain, DCT domain and color space conversions. Since pixel values are highly correlated in RGB color spaces, the use of YIQ color space for watermark embedding is beneficial for improvement in results. The PSNR and NC values for Q channel are better than PSNR and NC values for $\mathrm{Y}$ and I channels.

Authors [15] proposed an algorithm in RGB color space. The cover image is decomposed into three separate colors planes namely R, G and B. Individual planes are decomposed into sub bands using DWT. DCT is applied in HH component of each plane. Secret images are dispersed among the selected DCT coefficients using a pseudo random sequence and a Session key. They used only selected high frequency components are modified for the hiding method; therefore there must be a constraint on the secret image size

In [16], Color Image Watermarking algorithm based on DWT-SVD is proposed. The scrambling watermark is embedded into green component of color image based on DWT-SVD. The scheme is robust and giving PSNR up to $42.82 \mathrm{db}$

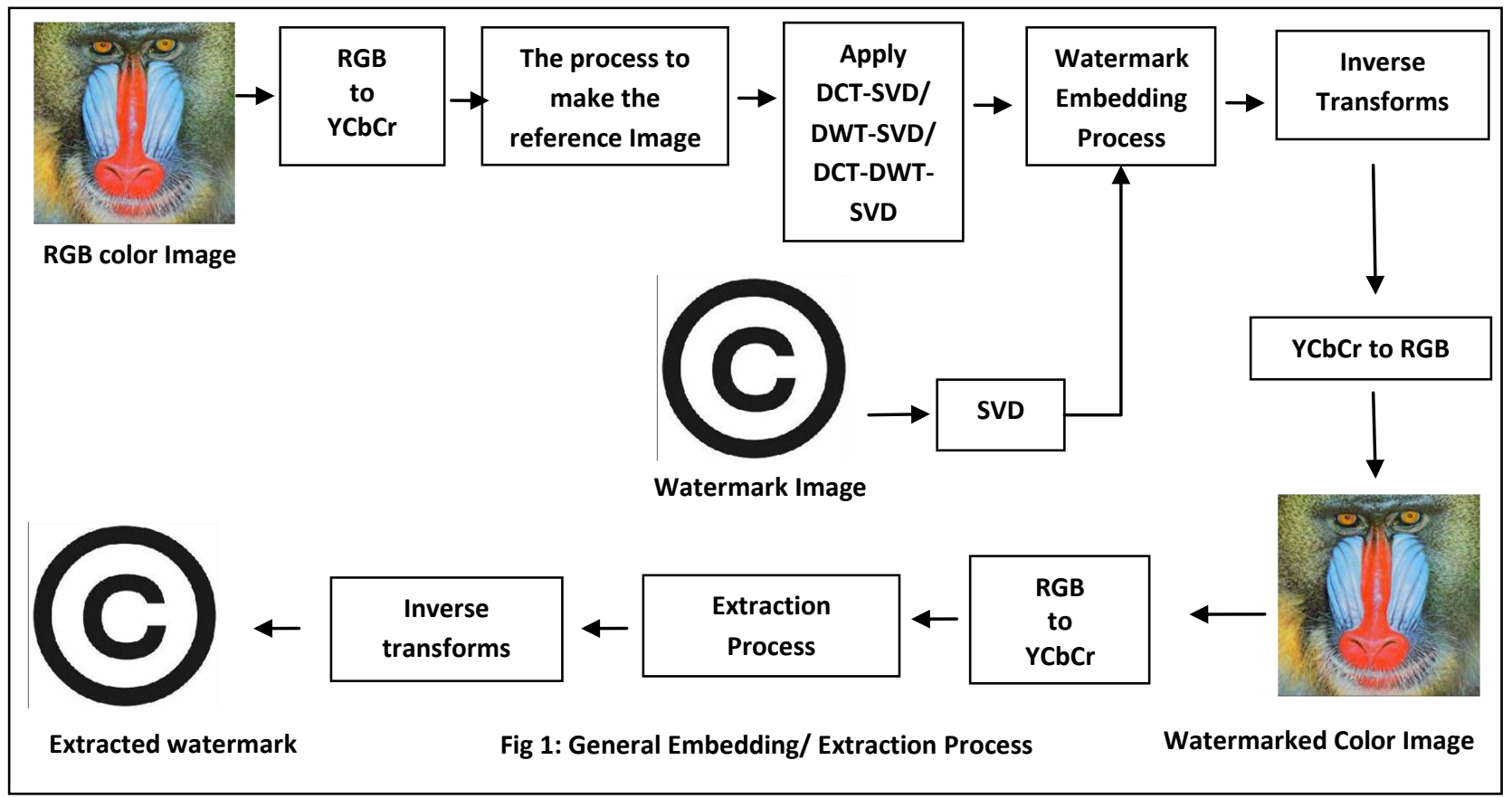

\section{PROPOSED ALGORITHMS}

The watermark embedding and extraction process has shown in figure 1 .

\subsection{Algorithm Using DCT-SVD}

Watermark Embedding Procedure

Step 1: Convert RGB image to $Y C_{b} C_{r}$ color matrix format. Step 2: The Y matrix is segmented into blocks of size $p_{1} \times$ $\mathrm{p}_{2}$ via ZIG_ZAG sequence denoted by $\mathrm{F}^{1}$, where 1 is the number of blocks.

Step 3: Find out the spatial frequency of all blocks, denoted by $\mathrm{SF}_{\mathrm{F}} \mathrm{l}$.
Step 4: Significant blocks are found out based on their spatial frequency. Spatial frequencies of each block are stored in descending order. Then make a threshold on spatial frequency. Those blocks, which have spatial frequency less than or equal to threshold, are considered as significant blocks and are used for making reference image, $f_{r e f}$ which is a size of $\mathrm{m} \times \mathrm{n}$.

Step 5: Perform DCT on the reference image, which is denoted by $f_{D C T}$

Step 6: Perform SVD transform on both $f_{D C T}$ and watermark image, denoted by $f_{W}$. 


$$
\begin{aligned}
& f_{d c t}^{S V D}=U_{f_{d c t}} * S_{f_{d c t}} * V_{f_{d c t}^{T}}^{T} \\
& f_{W}^{S V D}=U_{f_{W}} * S_{f_{W}} * V_{f_{W}}^{T}
\end{aligned}
$$

Step 7: Modify the single values of reference image with the singular values of watermark as

$$
\left(\sigma_{f_{r e f}}\right)^{*}=\sigma_{f_{d c t}^{S V D}}+\beta * \sigma_{f_{W}^{S V D}}
$$

Where $\beta$ give's the watermark strength.

Step 8: Perform inverse SVD,

$$
f_{i s v d}=U_{f_{d c t}} * S_{f_{r e f}}^{*} * V_{f_{d c t}^{T}}^{T}
$$

Step 9: Perform inverse DCT to construct the modified reference image, denoted by $f_{i d c t}$. Again $f_{i d c t}$ is segmented into blocks of size $\mathrm{p}_{1} \times \mathrm{p}_{2}$ and mapped onto their original positions for constructing the watermarked image, denoted by $F_{W}^{*}$

Step 10: Convert the watermark image from $Y C_{b} C_{r}$ to RGB color matrix.

\section{Watermark Extraction Procedure}

The objective of the watermark extraction is to obtain the estimate of the watermark. For watermark extraction, original reference and watermarked images, left and right singular vectors must be available at the receiver end.

Step 1: Convert the watermarked color image from RGB to $Y C_{b} C_{r}$ color matrix format.

Step 2: Using the positions of significant blocks, make the reference image from the watermarked Y matrix, denoted by $f_{\text {ref }}^{*}$

Step 3: Perform DCT on both $f_{\text {ref }}^{*}$ and original reference image, $f_{\text {reff }}$.

Step 4: Perform SVD transform on both DCT coefficients.

$$
\left(f_{D C T}^{S V D}\right)=U_{f_{D C T}}^{*} * S_{f_{D C T}}^{*} * V_{f_{D C T}}^{* T}
$$

Step 5: Extract the singular values of the watermark.

$$
\sigma_{W}^{E X T}=\frac{\sigma_{f_{D C T}}^{*}-\sigma_{f_{D C T}}^{*}}{\beta}
$$

Step 6: Obtain the extracted watermark as:

$$
W^{\text {ext }}=U_{W} * S_{W}^{\text {ext }} * V_{W}^{T}
$$

\subsection{Algorithm Using DWT-SCD}

\section{Watermark Embedding Procedure}

Step 1: Convert color image from $\mathrm{RGB}$ to $\mathrm{YC}_{\mathrm{b}} \mathrm{C}_{\mathrm{r}}$ color matrix format.

Step 2: The Y matrix is segmented into blocks of size $\mathrm{p}_{1} \times$ $\mathrm{p}_{2}$ via ZIG_ZAG sequence denoted by $\mathrm{F}^{\mathrm{l}}$, where 1 is the number of blocks.

Step 3: Find out the spatial frequency of all blocks, denoted by $\mathrm{SF}_{\mathrm{F}} \mathrm{l}$.

Step 4: Significant blocks are found out based on their spatial frequency. Spatial frequencies of each block are stored in descending order. Then make a threshold on spatial frequency. Those blocks, which have spatial frequency less than or equal to threshold, are considered as significant blocks and are used for making reference image, $f_{\text {ref }}$ which is a size of $m \times n$.
Step 5: Perform DWT on the reference image. It divides the image into four sub bands, LL, LH, HL and HH. Select the LL band, which is denoted by $f_{L L}$

Step 6: Perform SVD transform on both $\mathrm{f}_{\mathrm{LL}}$ and watermark image.

$$
\begin{aligned}
& f_{L L}^{S V D}=U_{L L} * S_{L L} * V_{L L}^{T} \\
& f_{W}^{S V D}=U_{W} * S_{W} * V_{W}^{T}
\end{aligned}
$$

Step 7: Modify the single values of reference image with the singular values of watermark as

$$
\left(\sigma_{f_{\text {ref }}}\right)^{*}=\sigma_{L L}+\beta * \sigma_{W}
$$

Here $\beta$ give's the watermark strength.

Step 8: Perform inverse SVD,

$$
f_{i S V D}^{*}=U_{L L} * S_{f_{r e f}}^{*} * V_{L L}^{T}
$$

Step 9: Perform inverse DWT to construct the modified reference image, denoted by $f_{D W T}^{*}$. Again $f_{D W T}^{*}$ is segmented into blocks of size $\mathrm{p}_{1} \times \mathrm{p}_{2}$ and mapped onto their original positions for constructing the watermarked image.

Step 10: Convert watermarked image from $Y C_{b} C_{r}$ to RGB color matrix.

Watermark Extraction Procedure

Step 1: Convert watermarked color image from RGB to $\mathrm{YC}_{\mathrm{b}} \mathrm{C}_{\mathrm{r}}$ color matrix format.

Step 2: Using the positions of significant blocks, make the reference image from the watermarked Y matrix, $f_{r e f}^{*}$.

Step 3: Perform DWT on both $f_{\text {ref }}^{*}$ and original reference image, $\mathrm{f}_{\mathrm{ref}}$.

Step 4: Perform SVD transform on both $f_{L L}$ and $f_{\text {ref }}^{W}$.

$$
\left(f_{L L}^{S V D}\right)^{*}=U_{L L}^{*} * S_{L L}^{*} * V_{L L}^{T}
$$

Step 5: Extract the singular values of the watermark.

$$
\sigma_{W}^{e x t}=\frac{\sigma_{L L}^{*}-\sigma_{f_{L L}}}{\beta}
$$

Step 6: Obtain the extracted watermark as:

$$
W^{\text {ext }}=U_{W} * S_{W}^{e x t} * V_{W}^{T}
$$

\subsection{Algorithm Using DWT-DCT-SCD}

Watermark Embedding Procedure

Step 1: Convert color image from $\mathrm{RGB}$ to $\mathrm{YC}_{\mathrm{b}} \mathrm{C}_{\mathrm{r}}$ color matrix format.

Step 2: The Y matrix is segmented into blocks of size $\mathrm{p}_{1} \times$ $\mathrm{p}_{2}$ via ZIG_ZAG sequence denoted by $\mathrm{F}^{\mathrm{l}}$, where 1 is the number of blocks.

Step 3: Find out the spatial frequency of all blocks, denoted by $\mathrm{SF}_{\mathrm{F}} \mathrm{l}$

Step 4: Significant blocks are found out based on their spatial frequency. Spatial frequencies of each block are stored in descending order. Then make a threshold on spatial frequency. Those blocks, which have spatial frequency less than or equal to threshold, are considered as significant blocks and are used for making reference image, $\mathrm{f}_{\mathrm{ref}}$ which is a size of $m \times n$. 
Step 5: Perform DWT on the reference image. It divides the image into four sub bands, LL, LH, HL and HH. Select the LL band, which is denoted by $f_{L L}$. Perform DCT on the $f_{L L}$, which is denoted by $f_{D C T}^{L L}$.

Step 6: Perform SVD transform on both $\epsilon$ and watermark image, W.

$$
\begin{gathered}
f_{D C T}^{L L, S V D}=U_{f_{D C T}} * S_{f_{D C T}} * V_{f_{D C T}}^{T} \\
W=U_{W} * S_{W} * V_{W}^{T}
\end{gathered}
$$

Step 7: Modify the single values of reference image with the singular values of watermark as

$$
\left(\sigma_{f_{\text {ref }}}\right)^{*}=\sigma_{f_{D C T}}+\beta * \sigma_{W}
$$

Where $\beta$ give's the watermark strength.

Step 8: Perform inverse SVD,

$$
f_{f S V D}^{*}=U_{f_{D C T}} * S_{f_{D C T}}^{*} * V_{f_{D C T}}^{T}
$$

Step 9: Perform inverse DCT denoted by $f_{i D C T}^{*}$. Now perform DWT on $f_{i D C T}^{*}$, which is denoted by $\mathrm{f}_{\mathrm{iDWT}}^{*}$ and is segmented into blocks of size $\mathrm{p}_{1} \times \mathrm{p}_{2}$ and mapped onto their original positions for constructing the watermarked image.

Step 10: Convert watermarked image from $\mathrm{YC}_{\mathrm{b}} \mathrm{C}_{\mathrm{r}}$ to $\mathrm{RGB}$ color matrix.

Watermark Extraction Procedure

Step 1: Convert watermarked color image from RGB to $\mathrm{YC}_{\mathrm{b}} \mathrm{C}_{\mathrm{r}}$ color matrix format..

Step 2: Using the positions of significant blocks, make the reference image from the watermarked $\mathrm{Y}$ matrix, $\mathrm{f}_{\text {ref }}^{*}$

Step 3: Perform DWT and DCT on both $\mathrm{f}_{\text {ref }}^{*}$ and original reference image, $\mathrm{f}_{\text {ref }}$.

Step 4: Perform SVD transform on both DCT coefficients.

$$
f_{r e f}^{W}=U_{f_{r e f}^{W}}^{W} * S_{f_{r e f}^{W}}^{W} * V_{f_{r e f}^{W}}^{T}
$$

Step 5: Extract the singular values of the watermark.

$$
\sigma_{W}^{e x t}=\frac{\sigma_{f_{r f}^{W}}-\sigma_{f_{D C T}}}{\beta}
$$

Step 6: Obtain the extracted watermark as:

$$
W^{\text {ext }}=U_{W} * S_{W}^{e x t} * V_{W}^{T}
$$

\section{EXPERIMENTAL RESULTS}

We evaluated the performance of proposed color image watermarking algorithms with mandrill and peppers of size $512 \times 512$. The watermark used in our experiment was a gray scale image. For DCT-SVD algorithm the watermark size was $256 \mathrm{x} 256$, for DWT-SVD and DWT-DCT-SVD algorithms watermark size was $128 \times 128$. This has shown in fig2 to fig9. We showed the results with mandrill image.

\subsection{Performance Measures}

\subsubsection{Imperceptibility Performance}

Imperceptibility means that the perceived quality of the color image should not be distorted by the presence of the watermark [9]. As a measure of the quality of a watermarked image, the peak signal to noise ratio (PSNR) is typically used.
In our work, the watermark was embedded in the color image according to the algorithms discussed in the section3. For the algorithm DCT-SVD the embedding depth of watermark was 0.035. The corresponding PSNR of the watermarked color image was 40.1516 dB. We used the watermark depth for DWT-SVD was 0.05. The corresponding PSNR value was 43.6499dB, while the embedding depth for DWT-DCT-SVD was 0.075 and the corresponding PSNR was $\mathbf{4 0 . 5 2 6 5}$

\subsubsection{Robustness Performance}

Robustness of a watermarking algorithm is a measure of the immunity or resistance of the watermark against attempts to remove or degrade it from the watermarked color image by different types of digital signal processing attacks [10]. The similarity between the original watermark and the extracted watermark from the attacked watermarked image was measured by using the correlation factor $\rho$, which is computed using the following Equation:

$\rho(w, \widetilde{w})=\frac{\sum_{i=1}^{N} w * \widetilde{w}}{\sqrt{\sum_{i=1}^{N} w^{2}} \sqrt{\sum_{i=1}^{N} \widetilde{w}^{2}}}$

Where $\mathrm{N}$ is the number of pixels in watermark, $\mathrm{w}$ and $\widetilde{W}$ is the original and extracted watermarks respectively. The correlation factor $\rho$, may take values between -1 and 1 . The $\rho$ values for DCT-SVD, DWT-SVD and DWT-DCT-SVD were $0.9937,0.9683$ and 0.9693 respectively.

\subsection{Result Analysis}

We evaluated robustness of the algorithms against the following image attacks: average filtering, median filtering, noise attacks (Gaussian and salt \& pepper), JPEG compression, and rotation, cropping, resize, histogram equalization, pixilated, sharpening and contrast. Important attacks on watermarked color image were average filtering and median filtering. We tried to mask the watermarked image by mask value with $13 \times 13$. This has shown in figure10 and figure11 respectively. We got good robustness value for this mask value. Two kinds of common attacks were Additive Gaussian and Salt \& pepper noise. Additive Gaussian noise was tested with zero mean and 0.75 variance. As shown in figure12. Salt \& pepper noise was tested with the value of 0.5 , has shown in fig21. These results generally indicate robustness of the proposed algorithm against addition of Gaussian and Salt and Pepper noise. The watermarked color image was compressed with a quality factor of 80:1. As shown in figure13 and the correlation value indicates clearly the high robustness of the proposed algorithm for compression attack. Another important attack was cropping. Here we cropped the watermarked image such that $25 \%$ area was reaming and shown figure14. The other prime image processing attack was resizing. The resizing was performed from 512-> 128-> 512 shown in figure15. The watermarked color image was rotated with an angle of $50^{\circ}$. As shown in figure16. Histogram equalization attack was shown in figure17. It showed that the proposed algorithms had high robustness against this attack. The pixilated, sharpen and contrast has shown figures 18,19 and 20 respectively. 


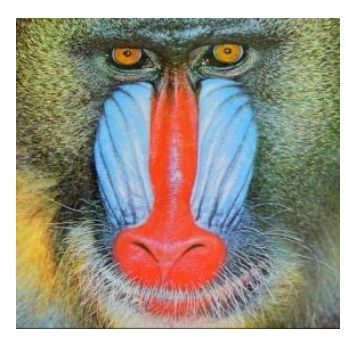

Fig2: Original mandrill

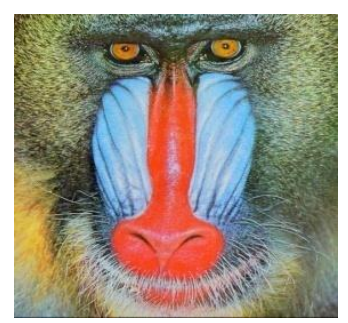

Fig4: watermarked

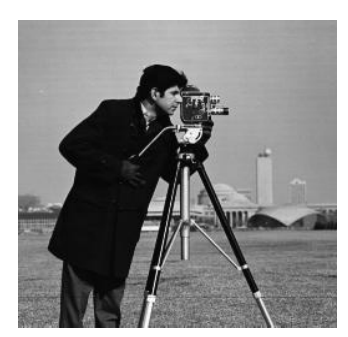

Fig3: Original Watermark

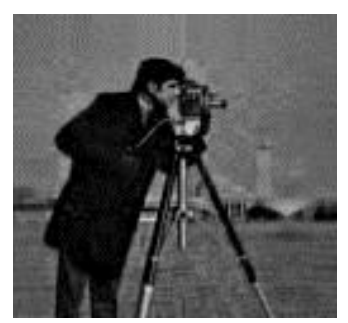

Fig5:Extracted watermark

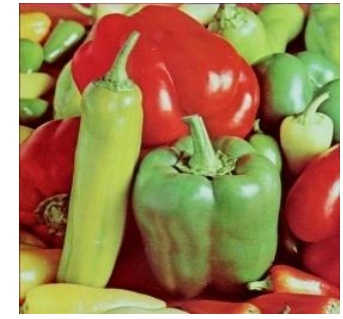

Fig6: Original peppers

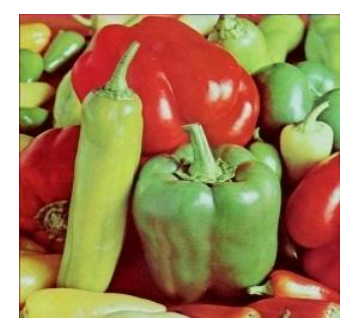

Fig8: watermarked

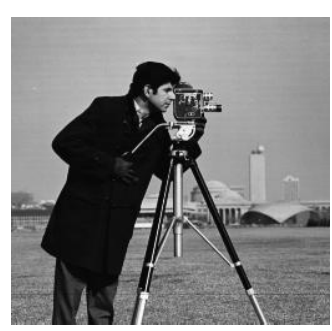

Fig7: Original Watermark

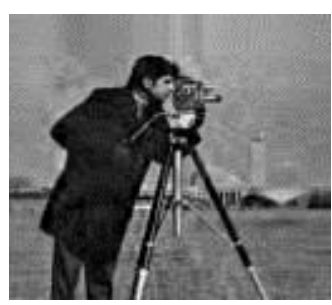

Fig9:Extracted watermark

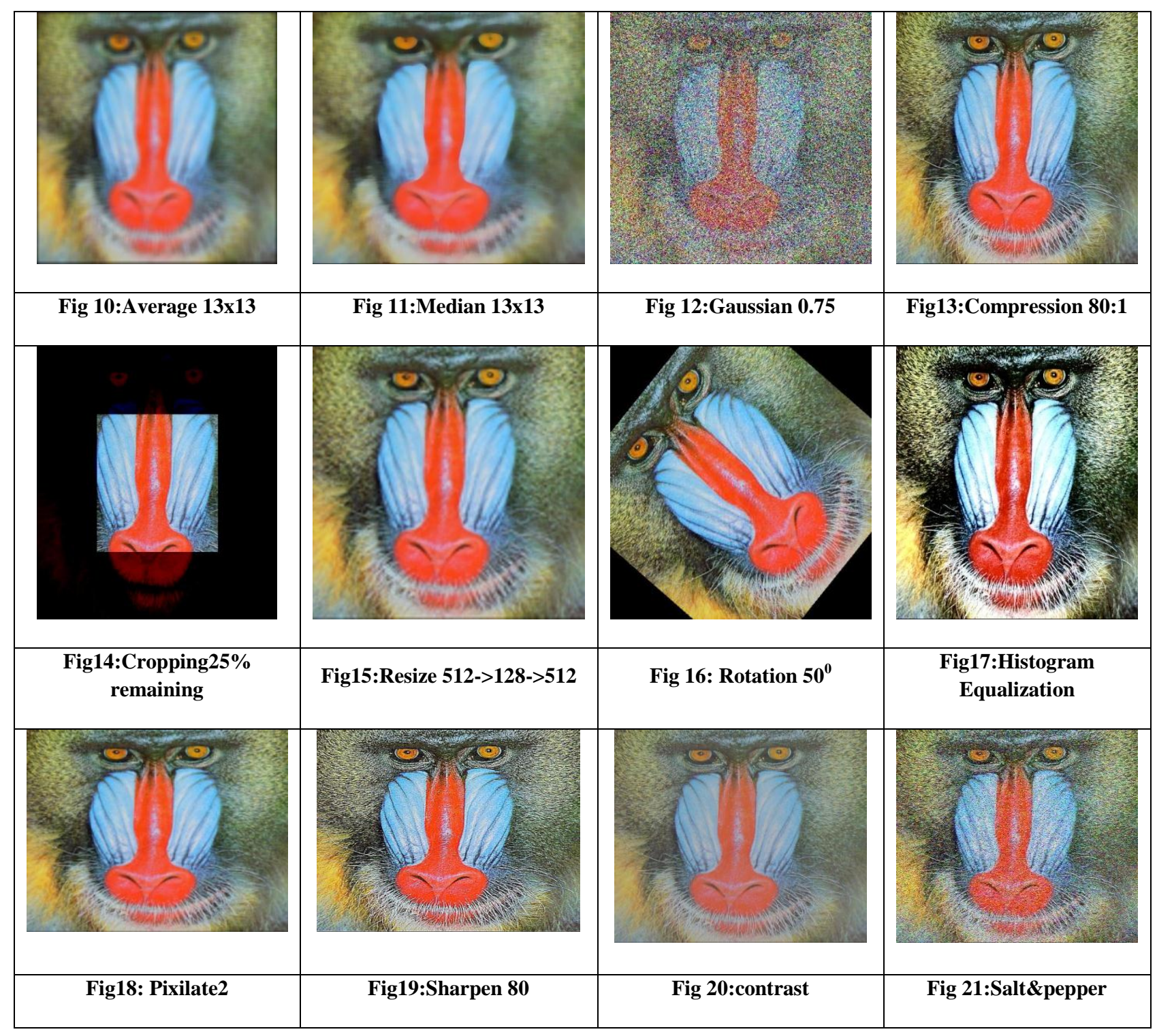




\begin{tabular}{|c|c|c|c|c|c|c|}
\hline \multirow{3}{*}{ Attacks } & \multicolumn{3}{|c|}{ Mandrill Image } & \multicolumn{3}{|c|}{ Peppers Image } \\
\hline & \multicolumn{2}{|c|}{ Existing Methods } & \multirow{2}{*}{$\begin{array}{c}\text { Proposed } \\
\text { Dct-Dct-Svd }\end{array}$} & \multicolumn{2}{|c|}{ Existing Methods } & \multirow{2}{*}{$\begin{array}{l}\text { Proposed } \\
\text { Dct-Dct-Svd }\end{array}$} \\
\hline & Dct-Svd & Dwt-Svd & & Dct-Svd & Dwt-Svd & \\
\hline $\begin{array}{l}\text { Average Filtering } \\
(13 \times 3)\end{array}$ & -0.1119 & -0.0881 & -0.0275 & -0.1682 & -0.2118 & -0.0949 \\
\hline $\begin{array}{l}\text { Median Filtering } \\
\quad(13 \times 13)\end{array}$ & -0.0098 & 0.1675 & 0.2505 & 0.0444 & 0.3929 & 0.4233 \\
\hline $\begin{array}{l}\text { Additive Gaussian } \\
\text { Noise }(75 \%)\end{array}$ & 0.0078 & -0.1188 & -0.1011 & 0.1010 & 0.2772 & 0.2952 \\
\hline $\begin{array}{c}\text { JPEG compression } \\
(80: 1)\end{array}$ & 0.9510 & 0.9610 & 0.9671 & 0.9910 & 0.9947 & 0.9946 \\
\hline $\begin{array}{l}\text { Cropping ( } 25 \% \text { area } \\
\text { remaining) }\end{array}$ & 0.1421 & -0.5449 & -0.3807 & 0.0339 & -0.7511 & -0.7462 \\
\hline $\begin{array}{c}\text { Resizing (512 -> } 128 \\
->512)\end{array}$ & 0.0050 & 0.1373 & 0.2308 & 0.2739 & 0.4714 & 0.6341 \\
\hline Rotation $\left(50^{\circ}\right)$ & -0.4310 & -0.3838 & -0.3771 & -0.5504 & -0.4855 & -0.4765 \\
\hline Pixilation 2 & 0.0190 & 0.0872 & 0.0872 & 0.4870 & 0.5632 & 0.7649 \\
\hline salt \& pepper & 0.0724 & -0.0285 & 0.0948 & 0.1364 & 0.2496 & 0.3366 \\
\hline $\begin{array}{l}\text { Histogram } \\
\text { equalization }\end{array}$ & 0.4669 & 0.4843 & 0.5106 & 0.6896 & 0.7484 & 0.7649 \\
\hline Sharpen80 & 0.1889 & 0.2239 & 0.2959 & 0.4870 & 0.6109 & 0.7580 \\
\hline Contrast(-50) & -0.5082 & -0.4138 & -0.3791 & -0.1531 & 0.0459 & 0.0897 \\
\hline & & ble-1 : & - $\quad<$ & values & & \\
\hline
\end{tabular}

\section{CONCLUSION}

In this paper we proposed three semi-blind reference watermarking algorithms. Those algorithms were DCT-SVD, DWT-SVD and DWT-DCT-SVD. The watermark was visually meaningful gray scale image. In these proposed algorithms DWT-SVD has good imperceptibility. In these proposed algorithms, the DCT-SVD was superior to the other two algorithms in additive Gaussian noise and cropping attacks. For remaining attacks the DWT-DCT-SVD algorithm has good robustness when compared with other two algorithms. Table 1 shows the NCC values of all the attacks.

\section{REFERENCES}

[1] JV. Potdar, S. Han and E. Chang, 2005. " A survey of Digital Image Watermaking Techniques", in Proceedings of the 2005 IEEE International Conference on Industrial Informatics,pp. 709-716.
[2] F. Hartung and M. Kutter, "Multimedia Watermaking Techniques," in Proceedings of the IEEE, vol. 87, no.7, pp. 1079-1107, July 1999.

[3] Chi-Kwong Chan , L.M. Cheng "Hiding data in images by simple LSB substitution "Pattern Recognition 37 (2004) 469-474,ww.elsevier.com/locate/patcog.

[4] Dr.M.A.Dorairangaswamy "A Robust Blind Image Watermaking Scheme in Spatial Domain for Copyright Protection" International Journal of Engineering and Technology Vol.1,No.3,August,2009,ISSN;1793-8236.

[5] Sanghyun Joo, Youngho Suh, Jacho Shin, and Hisakazu Kikuchi " A New Robust Watermark Embedding into Wavelet DC Components" ETRI Journal, Volume 24,Number5, October 2002.

[6] Gengming Zhu, and Nong Sang "Watermarking Algorithm Research and Implementation Based on DCT Block" Proceedings of world academy of 
science,engineering and technology volume 35 november 2008 issn 2070-3740.

[7] Ke-feng He,"Watermarking for images using the HVSand SVD in the wavelet domain" Proceedings of IEEEInternational Conference on Mechatronics and Automation,pp.2352-2356,2006.

[8] Liu Liang and Sun Qi, “ A new SVD-DWT composite watermarking,"ICSP proceedings of IEEE International conference on signal processing,2006.

[9] Voloshynovskiy, S.,S.Pereira, and T.Pun,2001. “ Attacks on Digital Watermarks: Classification,Estimation-Based Attacks, and Benchmarks". Communication Magazine 39,pp.118-126.

[10] Fabien A.P. Petitcoals,Ross J. Anderson,1999. "Evaluation of Copyright Marking Systems",IEEE International Conference on Multimedia Computing and Systems (ICMCS'99) 1,pp.574-579.

[11] C.Venkata Narasimhulu, K.Satya Prasad " A New SVD based Hybrid Color Image Watermarking for copyright Protection using Contourlet Transform" International Journal of Computer Applications (0975-8887), Volume 20-No.8,April 2011,pp.18-27.

[12] Tosihiro Akiyama,Fumiaki Motoyoshi, Osamu Uchida and Shohachiro Nakanishi "Hybrid Digital Watermarking For Color Images Based On Wavelet Transform" IADIS International Conference Applied Computing 2006,pp.548-551.

[13] R.Eswaraiah,Sai Alekhya Edara, E.Sreenivasa Reddy "Color Image Watermarking Scheme using DWT and DCT Coefficients of R,G and B Color omponents"International Journal of Computer applications (0975-8887) Volume 50-No.8,July 2012,pp.38-41.

[14] Baisa L.Gunjal, and Suresh N.Mali "Secured Color Image Watermarking Technique In Dwt-Dct Domain" International Journal of Computer Science, Engineering and Information Technology (IJCSEIT), Vol.1,No.3,August 2011,pp.36-44.

[15] Tanmay Bhattacharya,Nilanjan Dey, S.R.Bhadra Chaudhuri " A Session based Multiple Image Hiding Technique using DWT and DCT" International Journal of Computer Applications (0975-8887) Volume 38No.5,January 2012,pp.18-21.

[16] Cheng-qun Yin,Li Li An-qiang Lv and Li Qu,"Color Image Watermarking Algorithm Based on DWT-SVD", Proceeding of the IEEE International onfeence on Autimation and Logistics, August 18-21, 2007,Jinan,China,PP 2607-2611.

\section{Biographies}

P. Satyanarayana Murty is currently working as Sr.Associate Professor \& Head of the depatment in ECE Department, GIITS, Engineering College,Vishakapatnam, Andhra Pradesh, India. He is working towards his Ph.D.at AU College of Engineering, Vishakapatnam, India. He received his M.Tech from Jawaharlal Nehru Technological University, Hyderabad, India. He has fifteen years experience of teaching undergraduate students and post graduate students. His research interests are in the areas of image watermarking, and image compression

Dr. P. Rajesh Kumar is currently Associate Professor in ECE Department, AU College of Engineering,Vishakapatnam, India. He received his M.Tech and Ph.D.from AndhraUniversity, Vishakapatnam, India. He has eleven years experience of teaching undergraduate and postgraduate students and guided number of post-graduate theses. He has published 10 research papers in National and International journals. Presently he is guiding six Ph.D students in the area of digital signal processing and Image processing. His research interests are in the areas of digital image processing and digital signal processing. 